Research Article

\title{
FTIR Spectrophotometry as a Green Tool for Quantitative Analysis of Drugs: Practical Application to Amoxicillin
}

\author{
Stefany Fanelli, ${ }^{1}$ Alexander Zimmermann, ${ }^{2}$ Eliane Gandolpho Totóli $\left(\mathbb{D},{ }^{1}\right.$ \\ and Hérida Regina Nunes Salgado (D) $^{1}$ \\ ${ }^{1}$ School of Pharmaceutical Sciences, Universidade Estadual Paulista, Araraquara, SP, Brazil \\ ${ }^{2}$ Institute of Biochemistry and Biotechnology, Faculty of Natural Science I, Martin-Luther-University Halle-Wittenberg, \\ Halle (Saale), Germany
}

Correspondence should be addressed to Eliane Gandolpho Totóli; eliane.totoli@gmail.com

Received 14 May 2018; Accepted 19 September 2018; Published 23 October 2018

Academic Editor: Serkos A. Haroutounian

Copyright (C) 2018 Stefany Fanelli et al. This is an open access article distributed under the Creative Commons Attribution License, which permits unrestricted use, distribution, and reproduction in any medium, provided the original work is properly cited.

Amoxicillin is an antimicrobial agent that belongs to the penicillin compounds. Its bactericidal action causes a destruction of the cell wall of bacteria. It is widely used in clinical practice, and it belongs to the Brazilian National List of Essential Drugs (RENAME). In literature, there are some green analytical methods for the amoxicillin analysis; however, none of them is focused on its quantification in capsules. Therefore, the aim of this study was to validate an environmentally friendly analytical method for the analysis of this antimicrobial action in capsules, using spectrophotometry in the mid-infrared region. The analyses were performed in the spectral range of $1815-1736 \mathrm{~cm}^{-1}$, and the samples were analyzed as potassium bromide pellets. The method was validated according to the ICH guidelines and Brazilian legislation. Linearity, selectivity, precision, accuracy, and robustness were evaluated and showed adequate results for method validation, in a concentration range of $0.5-1.5 \mathrm{mg} / \mathrm{pellet}$. Thus, it is concluded that the validated spectrophotometric method is able to quantify amoxicillin in capsules. In addition, it is a fast, economical, and environmentally friendly method, since it does not use organic solvents, and it can be used for quality control of routine analysis of this drug.

\section{Introduction}

Amoxicillin is a broad-spectrum antimicrobial agent belonging to the class of penicillins and is widely used in clinical practice. Furthermore, it belongs to the Brazilian National List of Essential Drugs (RENAME). Amoxicillin is available in tablets, capsules, and powder for oral suspension. It is also available associated with potassium clavulanate, for the treatment of infections caused by $\beta$-lactamase-producing bacteria, and presented in the pharmaceutical dosage forms of tablets and oral suspension [1].

This penicillin is generally indicated for treating infections caused by microorganisms responsible for gonorrhea, pneumonia, bronchitis, otitis, or endocarditis. Furthermore, it is applied after infections of the urinary tract caused by Haemophilus influenzae. It can be used as an alternative treatment in cases of gastroenteritis and typhoid fever caused by Shigella. Amoxicillin is considered the drug of choice for the prophylaxis of bacterial endocarditis, and it can be combined with omeprazole and clarithromycin for combating Helicobacter pylori in the patients with peptic ulcer [2].

This drug has a rapid bactericidal action, acting on the cell wall of bacteria. It is very well-absorbed orally, and 60 to $70 \%$ are excreted renally. Generally, amoxicillin is administered three times daily as a $500 \mathrm{mg}$ capsule, or even twice daily, and it is well tolerated by the organism. However, it may have some undesirable effects such as nausea, vomiting, and gastrointestinal irritation [3].

In literature, there are several published studies describing the development of the analytical methods for analysis of amoxicillin in different matrices. However, most of the present methods use highly potential toxic solvents for the operators and environment, for example, 
high-performance liquid chromatography (HPLC) [4-8], ultraperformance liquid chromatography (UPLC) [9], or iodometry [10].

The quality of drugs has always been a concern of the World Health Organization (WHO). Since without the guarantee these products meet the required standards of quality, safety, and effectiveness, any health service is evidently affected [11]. Because of this, attention on the quality control of drugs is important. Pharmaceutical companies must ensure that their marketed products have adequate safety and efficacy for the population.

Therefore, the validation of the efficient analytical methods for use in the quality control of the marketed drugs is essential. The validation of an analytical methodology aims at verifying whether a tested method is suitable for a particular purpose, in other words, whether the method is able to analyze qualitatively and/or quantitatively a drug or related substances in pharmaceutical dosage forms [12-14].

The trend is that industries seek ways to reduce the impacts of their activities on the environment. Thus, they can adopt the position of reduction, prevention, or elimination of process waste. In this regard, there are some actions that can be taken, such as the replacement of the analytical methods that employs a high amount of organic solvents with others that do not use them, the replacement of a synthesis process by a greener one, or the exchange of raw materials or inputs by other less toxic ones [15].

In this context, the infrared spectrophotometry stands out. This method allows a quantification of substances without using organic solvents. This technique does not require any extraction step of the sample and therefore can also be used for substances with solubility problems.

Considering the importance of amoxicillin for clinical practice and the relevance of seeking green methods for the analysis of drugs, this work describes the development and validation of an innovative and environmental friendly analytical method, by spectrophotometry in the midinfrared region, for quantification of amoxicillin in capsules.

\section{Experiment}

2.1. Equipment. For spectrophotometric analysis, a spectrophotometer FTIR Shimadzu (Kyoto, Japan), IR Prestige21 model, was used. This equipment was connected to a computer to use the "IR Solution" software for analysis of the spectra. The construction of calibration curves was performed using Microsoft Excel (2013).

The other equipment used is as follows: H51 analytical balance (Mettler Toledo ${ }^{\mathrm{TM}}$, Barueri, Brazil) and oven ECB 1.2 Digital (Odontobrás ${ }^{\mathrm{TM}}$, Ribeirão Preto, Brazil).

2.2. Chemicals and Reagents. The pharmaceutical company União Química (São Paulo, Brazil) kindly donated amoxicillin reference standard (AMX RS) (declared a purity of 98.9\%) and amoxicillin (AMX) in the pharmaceutical dosage form of the capsule (Uni $\mathrm{Amox}^{\mathrm{TM}}, 500 \mathrm{mg} / \mathrm{capsule}$, União Química, São Paulo, Brazil). Adjuvants present in this pharmaceutical dosage form are sodium lauryl sulfate, croscarmellose sodium, and magnesium stearate. These substances were purchased from Sigma-Aldrich (São Paulo, Brazil). Potassium bromide ( $\mathrm{KBr}$ ) analytical grade was also used (Synth ${ }^{\mathrm{TM}}$, Diadema, Brazil).

2.3. Qualitative Analysis. To perform the method, the spectrophotometer described in Equipment was used. For the preparation of AMX RS pellets, an equivalent to $2.0 \mathrm{mg}$ of AMX RS was accurately weighted and then homogenized with $148.0 \mathrm{mg}$ of $\mathrm{KBr}$, previously powdered, and dried at $105^{\circ} \mathrm{C}$ in an oven to constant weight, in order to obtain a $150 \mathrm{mg}$ pellet (at $2 \mathrm{mg} /$ pellet). This mixture was compressed by a mechanical press for 10 minutes to obtain a translucent pellet. The spectral region included in the analysis was from 4000 to $400 \mathrm{~cm}^{-1}$ (the mid-infrared region). The analysis was held in transmittance, and the spectrum was obtained with the aid of "IR Solution" software (Shimadzu, Kyoto, Japan). The same procedure was performed with amoxicillin in capsules. Finally, a comparison of the spectra obtained from AMX RS and AMX capsule was performed to verify the similarity between them.

In addition, three independent pellets were prepared, containing each adjuvant present in the pharmaceutical dosage form (croscarmellose sodium, sodium lauryl sulfate, and magnesium stearate), in a concentration of $1.0 \mathrm{mg} /$ pellet. For this, $1.0 \mathrm{mg}$ of each component was homogenized with $149.0 \mathrm{mg}$ of $\mathrm{KBr}$. Each mixture was compressed by a mechanical press for 10 minutes to obtain translucent pellets. The spectral region included in the analysis was from 4000 to $400 \mathrm{~cm}^{-1}$ (the mid-infrared region). This procedure was performed to define the best spectral region to be used in the quantitative analysis (a region without interference of adjuvants).

\subsection{Quantitative Analysis}

2.4.1. Obtaining the Calibration Curve. To perform the method, the spectrophotometer described in "Equipment" section was used. Samples were analyzed as $\mathrm{KBr}$ pellets containing the drug. After obtaining the spectrum in the midinfrared range $\left(4000-400 \mathrm{~cm}^{-1}\right)$, the spectral region from 1815.0 to $1736.0 \mathrm{~cm}^{-1}$ was selected, which corresponds to a characteristic band of the amoxicillin molecule (carbonyl), and its height was quantitatively analyzed in absorbance.

During preliminary tests, five concentrations of amoxicillin were selected for the analytical curve, as follows: 0.5 , $0.75,1.0,1.25$, and $1.50 \mathrm{mg} /$ pellet.

For the preparation the pellets, the amounts equivalent to $0.50,0.75,1.00,1.25$, and $1.50 \mathrm{mg}$ of AMX RS (previously diluted in potassium bromide of $1: 10, \mathrm{w} / \mathrm{w}$ ) were taken and diluted with sufficient amounts of $\mathrm{KBr}$ to obtain $150 \mathrm{mg}$ pellets. The powders were mixed and ground to obtain a homogeneous mixture. Thereafter, this mixture was compressed with a mechanical press for 10 minutes to obtain translucent pellets. 
2.4.2. Determination of Amoxicillin in the Pharmaceutical Dosage Form. Amoxicillin content in capsules was calculated by Equation (1), and its percentage content was calculated by Equation (2):

$$
\begin{aligned}
C_{\mathrm{s}} & =A_{\mathrm{s}} \times \frac{C_{\mathrm{RS}}}{A_{\mathrm{RS}}}, \\
C_{\mathrm{s}} \% & =C_{\mathrm{s}} \times \frac{100}{C_{\mathrm{t}}} .
\end{aligned}
$$

where $C_{s}$ is the concentration of AMX in the sample (mg/pellet), $A_{s}$ is the absorbance of the AMX sample pellet, $C_{\mathrm{RS}}$ is the concentration of AMX RS solution (mg/pellet), $A_{\mathrm{RS}}$ is the absorbance of the AMX RS pellet, $C_{\mathrm{s}} \%$ is the percentage concentration of AMX in the sample, and $C_{t}$ is the theoretical concentration of AMX in the sample (mg/pellet)

2.4.3. Method Validation. The method was validated by analyzing the following parameters: linearity, precision, accuracy, robustness, and selectivity, as recommended by ICH guidelines and Brazilian legislation $[12,13]$. Limits of detection and quantification are not required for the validation of this class of the analytical method (Class I), according to ICH guidelines and Brazilian legislation $[12,13]$.

(1) Linearity. To check the linearity of the method, five concentrations of AMX RS ( 0.5 to $1.5 \mathrm{mg} /$ pellet) were used and evaluated on three different days. Linearity was confirmed by linear regression of least squares and statistical analysis by ANOVA.

(2) Precision. Precision was evaluated in two different ways: by intermediate precision (interday and between analysts) and repeatability (intraday). Interday precision was performed by analysis of six pellets of AMX RS (at $1.0 \mathrm{mg} / \mathrm{pellet)}$ on three different days, at the same experimental conditions. Similarity between the absorbances obtained on different days was evaluated by analysis of variance (ANOVA). Between-analyst's precision was carried out by the analysis of six pellets of AMX RS (at $1.0 \mathrm{mg} /$ pellet) performed by two different analysts. In this case, the absorbances were compared by $F$-test and $t$-test. Regarding intraday precision, it was evaluated by the analysis of six consecutive pellets of AMX RS (at $1.0 \mathrm{mg} / \mathrm{pellet)} \mathrm{in} \mathrm{the} \mathrm{same} \mathrm{day} \mathrm{and} \mathrm{at} \mathrm{the} \mathrm{same}$ working conditions. Thereafter, the percentage relative standard deviation value (RSD) between the absorbances was calculated [13].

(3) Accuracy. Accuracy was performed by the analysis of AMX RS recovery, in triplicate, at three levels (R1, R2, and R3), from 80 to $120 \%$ of the working concentration of the method (1.0 mg/pellet). The pellets for the recovery assay are prepared in accordance with the description in Table 1.

The recovery percentage was calculated by the equation determined by the Association of Official Analytical Chemists (AOAC) [16].
(4) Robustness. Robustness aims at showing the reliability of the method after small variations in the analytical parameters. The following parameters were varied: temperature of the workroom $\left(21^{\circ} \mathrm{C}\right.$, with the air conditioning on, and $26 \mathrm{C}$, with the air conditioning off), compression time of the pellets ( 2 minutes above and below the working compression time), and $\mathrm{KBr}$ brand (Shimadzu). For this, six pellets of AMX RS (at $1.0 \mathrm{mg} /$ pellet) were analyzed under each previously described condition. Similarity of the results was evaluated by the $F$-test and $t$-test, comparing the normal working conditions.

(5) Selectivity. Selectivity was analyzed in order to verify the ability of the method to quantify the drug in the presence of the adjuvants present in the pharmaceutical dosage form of the capsule $[12,13]$. The used method has been previously described in Qualitative analysis.

\section{Results}

3.1. Qualitative Analysis. Figure 1 shows an overlay of the absorption spectra of the AMX RS and sample. Figure 2 displays the absorption spectra of each adjuvant present in the capsules. The spectral range chosen for quantitative analysis (from 1815 to $1736 \mathrm{~cm}^{-1}$ ) is also identified in both figures.

\subsection{Quantitative Analysis}

3.2.1. Linearity. Three calibration curves, generated on three different days, were analyzed by a graphical representation. The average values of absorbance were plotted against their respective concentrations. A final analytical curve was obtained by averaging the three analytical curves, resulting in a correlation coefficient $(r)$ of 0.9971 , as shown in Figure 3.

ANOVA calculated for the data of the analytical curve showed an $F_{\text {calculated }}$ of 521.45 and $F_{\text {critical }}$ of 0.00445 .

3.2.2. Precision. Precision was evaluated according to the repeatability (intraday precision) and intermediate precision (interday precision) and between analysts. Intraday precision shows a RSD of $3.80 \%$ among the absorbance values. Table 2 shows the absorbances obtained for the evaluation of interday precision and between analysts.

The absorbances obtained for interday precision (Table 2) were compared by ANOVA. F calculated and $F_{\text {critical }}$ were 1.94 and 3.68 , respectively. To calculate the precision between analyst, $F$-test and $t$-test were used (Table 3 ). As the result, $t_{\text {calculated }}$ and $t_{\text {critical }}$ were 1.94 and 2.23 , respectively, showing no significance.

3.2.3. Accuracy. Accuracy was evaluated by the recovery test and conducted at three different concentration levels, and the results are shown in Table 3.

3.2.4. Robustness. Robustness was evaluated by small variations in working parameters, such as the temperature of the 
TABLe 1: Preparation of pellets for the recovery assay of the method of FT-IR spectrophotometry for amoxicillin.

\begin{tabular}{lcccc}
\hline & $\begin{array}{c}\text { AMX sample }(\mathrm{mg})(\text { diluted } 1: 10 \\
\text { w/w in KBr) }\end{array}$ & $\begin{array}{c}\text { AMX RS (mg) (diluted 1:10 } \\
\text { w/w in KBr) }\end{array}$ & $\begin{array}{c}\text { Amount of KBr } \\
(\mathrm{mg}))^{1}\end{array}$ & $\begin{array}{c}\text { Final theoretical concentration } \\
(\mathrm{mg} / \mathrm{pellet})\end{array}$ \\
\hline Sample & 5.0 & - & 145.0 & 0.50 \\
R1 & 5.0 & 3.0 & 142.0 & 0.80 \\
R2 & 5.0 & 5.0 & 140.0 & 1.00 \\
R3 & 5.0 & 7.0 & 138.0 & 1.20 \\
Reference & - & 5.0 & 145.0 & 0.50 \\
standard & & &
\end{tabular}

${ }^{1}$ Sufficient amount for the preparation of pellets with a total weight of $150 \mathrm{mg}$. AMX: amoxicillin; AMX RS: amoxicillin reference standard.

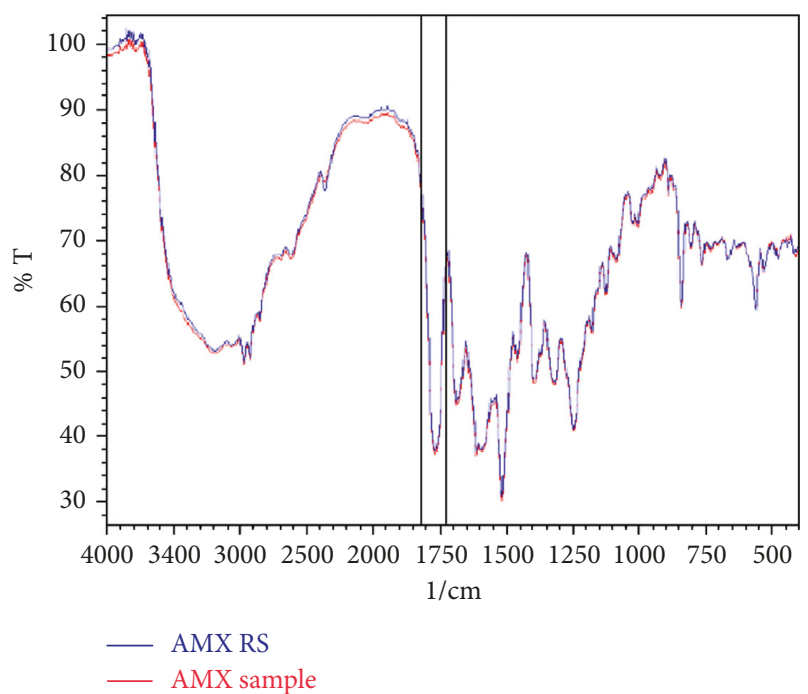

FIGURE 1: Overlap of the absorption spectra in the mid-infrared region of AMX RS and in the pharmaceutical dosage form of capsules. AMX RS: amoxicillin reference standard; AMX: amoxicillin. Spectral range chosen for quantitative analysis (from 1815 to $1736 \mathrm{~cm}^{-1}$ ) is highlighted.

workroom, pellet compression time, and $\mathrm{KBr}$ brand. The obtained experimental values were evaluated by $F$-test and $t$-test, by comparing the absorbance values from the normal and varied conditions. The obtained absorbances are presented in Table 4.

For the temperature of the workroom, the values of $t_{\text {calculated }}$ and $t_{\text {critical }}$ were 4.96 and 2.36 , respectively. This result is statistically significant.

Regarding the pellet compression time, the absorbances obtained for the normal working condition (10 minutes) were compared, individually, with each varied condition (8 and 12 minutes). The comparison between 8 and 10 minutes showed $t_{\text {calculated }}$ and $t_{\text {critical }}$ of 2.04 and 2.26, respectively. This result is statistically significant. In relation to the higher pellet compression times comparison (10 and 12 minutes), the $t_{\text {calculated }}$ was 1.97 and $t_{\text {critical }} 2.31$, showing no significance.

Concerning the $\mathrm{KBr}$ brand, the absorbances obtained from two different brands were compared (Synth and Shimadzu). In this case, the values of $t_{\text {calculated }}$ and $t_{\text {critical }}$ were 1.21 and 2.23, respectively, showing no significance.

3.2.5. Selectivity. Results obtained from selectivity analysis have been previously described in Qualitative analysis.
Figure 1 presents an overlay of the absorption spectra of the AMX RS and sample. Figure 2 presents the absorption spectra of each adjuvant present in the analyzed pharmaceutical dosage form. The spectral range chosen for quantitative analysis (from 1815 to $1736 \mathrm{~cm}^{-1}$ ) is also identified in both figures.

3.3. Determination of Amoxicillin in the Pharmaceutical Dosage Form. Three quantifications of amoxicillin in the capsules were performed, according to Equations (1) and (2). Table 5 shows the results of these assays.

\section{Discussion}

Environmental preservation is an important issue nowadays and is increasingly a concern of chemical and pharmaceutical industries. In order to reduce the environmental damage caused by toxic waste generation, companies look for alternatives to reduce, prevent, and even eliminate the generation of chemical waste from their processes. One option is to replace methods that use organic solvents by others that do not use the environmentally friendly methods $[15,17]$.

It is in this context, the infrared spectrophotometry stands out because it is a method that allows to quantify substances without organic solvents. It is suitable for drugs with solubility problems, since they can be analyzed in the solid form [18]. Infrared spectrophotometry is based on the fact that the chemical bonds of the molecules have natural vibrational frequencies. Each molecule only absorbs selected frequencies of radiation in the infrared region, which are equivalent to its natural vibrational frequencies. This absorption increases the amplitude of vibrational motion of the chemical bonds. Thus, the frequency of vibration may be associated with a particular type of the band $[19,20]$.

In addition to the absence of organic solvents, the spectrophotometric method in the infrared region has other advantages, such as being a rapid technique that does not require a pretreatment of the sample and assisting in detecting impurities. Another advantage is the low cost of materials for the manufacture of pellets. On the other hand, this method requires high control of humidity and temperature of the working environment.

The development of the analytical methods by spectrophotometry in the mid-infrared region has been successfully used in the quantification of other drugs [18, 21-36]. 

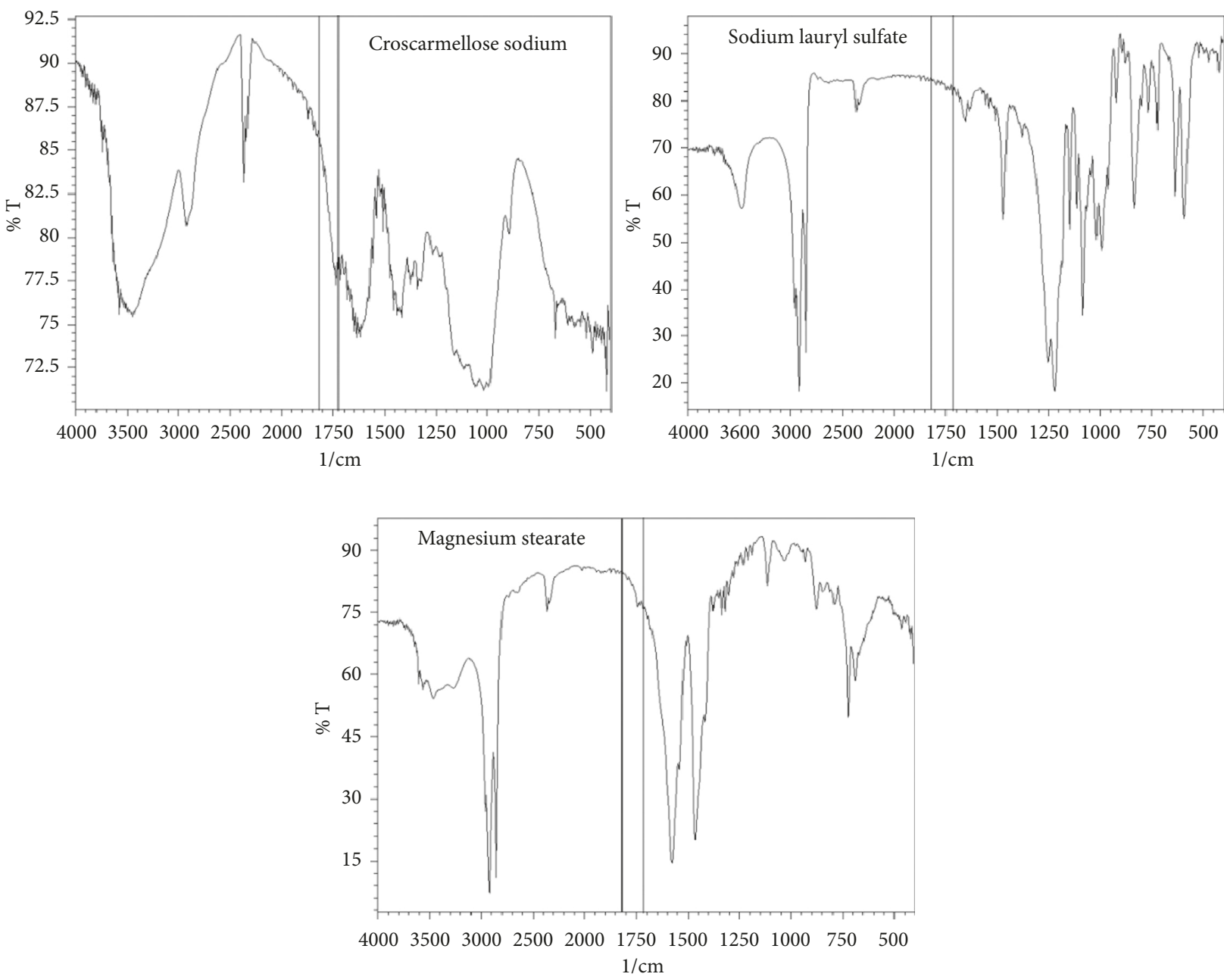

FIGURE 2: Absorption spectra in the mid-infrared region of the adjuvants present in the pharmaceutical dosage form of the capsule. Spectral range chosen for quantitative analysis (from 1815 to $1736 \mathrm{~cm}^{-1}$ ) is highlighted.

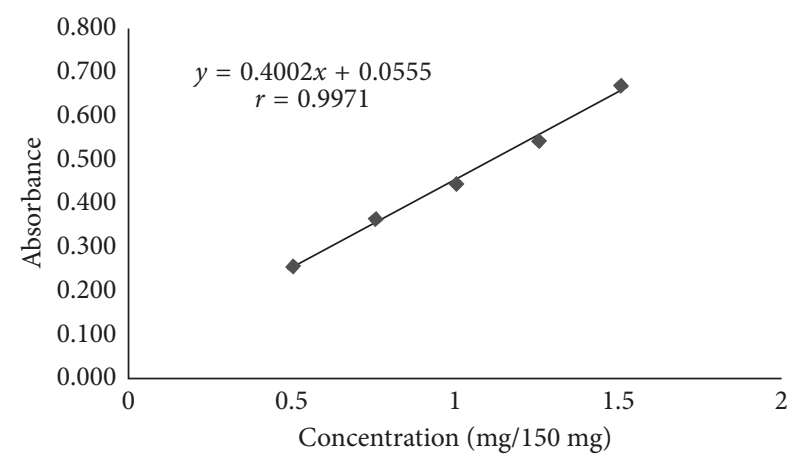

FIGURE 3: Graphical representation of the amoxicillin analytical curve by FT-IR spectrophotometry.

To develop the proposed method, the first step was a qualitative test. For this, an overlap of the spectra obtained with amoxicillin reference standard and amoxicillin in capsules was carried out (Figure 1). It was noticeable that both spectra have the same absorption bands, confirming the absence of adjuvants interference, impurities, or degradation products. The similarity between the spectra is a strong indicative of the identity of amoxicillin in the pharmaceutical dosage form.

The second step for the development of the quantitative method was to select a spectral range in the spectrum of amoxicillin in the mid-infrared region, to be quantitatively analyzed. For this, a comparison between the spectrum obtained with amoxicillin reference standard and each adjuvant present in the composition of amoxicillin in capsules was performed (Figure 2). It was observed that, between 1815 and $1736 \mathrm{~cm}^{-1}$, which represents the amoxicillin carbonyl band, there was no adjuvants interference. Thus, this was the spectral range selected for the quantitative analysis.

After the selection of the spectral range for the quantitative analysis, the analytical curve was constructed. With this purpose, many concentrations of AMX RS were tested, and five of them were selected for the method, as follows: 0.5 , $0.75,1.0,1.25$, and $1.50 \mathrm{mg} /$ pellet. Thereafter, the method was validated according to the ICH guidelines and Brazilian legislation $[12,13]$.

Three calibration curves, generated on three different days, were analyzed by a graphical representation. The average values of absorbance were plotted against their 
TABLE 2: Absorbances obtained for the evaluation of interday and between analysts precision to validate the spectrophotometric method in the mid-infrared region for the analysis of amoxicillin capsules.

\begin{tabular}{lcccc}
\hline & Interday & & \multicolumn{2}{c}{ Between analysts } \\
Day 1 & Day 2 & Day 3 & Analyst 1 & 0.466 \\
Analyst 2 \\
\hline 0.466 & 0.447 & 0.424 & 0.472 & 0.447 \\
0.472 & 0.417 & 0.456 & 0.458 & 0.413 \\
0.458 & 0.413 & 0.445 & 0.437 & 0.445 \\
0.437 & 0.445 & 0.436 & 0.436 & 0.447 \\
0.432 & 0.447 & 0.417 & 0.432 & 0.417 \\
\hline
\end{tabular}

TABle 3: Accuracy of the spectrophotometric method in the mid-infrared region for analysis of amoxicillin capsules.

\begin{tabular}{|c|c|c|c|c|c|}
\hline & Theoretical concentration (mg/pellet) & Real concentration (mg/pellet) & Recovery (\%) & RSD \% & Average recovery $(\%)$ \\
\hline R1 & 0.800 & 0.798 & 99.800 & 1.45 & \\
\hline $\mathrm{R} 2$ & 1.000 & 1.010 & 101.040 & 0.66 & 100.420 \\
\hline R3 & 1.200 & 1.205 & 100.420 & 0.80 & \\
\hline
\end{tabular}

RSD: relative standard deviation.

TABLE 4: Absorbances obtained during the robustness evaluation of the spectrophotometric method in the mid-infrared region for the analysis of amoxicillin capsules.

\begin{tabular}{cccccc}
\hline \multicolumn{2}{c}{$\begin{array}{c}\text { Temperature of } \\
\text { workroom }\end{array}$} & \multicolumn{2}{c}{ Pellet compression time } & \multicolumn{2}{c}{ KBr brand } \\
$21^{\circ} \mathrm{C}^{*}$ & $26^{\circ} \mathrm{C}$ & $8 \mathrm{~min}$ & $10 \mathrm{~min}^{*}$ & 12 min & Synth $^{*}$ \\
\hline 0.472 & 0.495 & 0.415 & 0.428 & 0.435 & 0.430 \\
0.447 & 0.494 & 0.420 & 0.424 & 0.434 & 0.433 \\
0.460 & 0.504 & 0.418 & 0.427 & 0.432 & 0.435 \\
0.458 & 0.508 & 0.417 & 0.417 & 0.413 & 0.434 \\
0.447 & 0.491 & 0.422 & 0.421 & 0.434 & 0.439 \\
0.491 & 0.503 & 0.413 & 0.417 & 0.433 & 0.433 \\
\hline
\end{tabular}

${ }^{*}$ Normal working condition.

TABLE 5: Determination of amoxicillin in the capsules.

\begin{tabular}{lccc}
\hline Assay & AMX content $(\mathrm{mg} /$ capsule) & AMX content $(\%)$ & Average content (\%) \\
\hline 1 & 501.535 & 100.307 & 100.340 \\
2 & 501.150 & 100.230 & 0.122 \\
3 & 502.350 & 100.470 & \\
\hline
\end{tabular}

AMX: amoxicillin; RSD: relative standard deviation.

respective concentrations. A final analytical curve was obtained by averaging the three analytical curves, resulting in a correlation coefficient $(r)$ of 0.9971 , as shown in Figure 3. The $r$ value is situated in accordance with the required $r$ values determined by the validation guidelines in the area of acceptance. Statistical analysis (ANOVA) of the results showed adequate regression of the calibration curve, as the $F_{\text {calculated }}(521.45)$ was higher than $F_{\text {critical }}(0.00445)$.

Precision was evaluated according to the repeatability (intraday precision) and intermediate precision (interday) and between analysts. The intraday precision showed adequate response, as the calculated RSD between the absorbances obtained from six samples, analyzed at the same day and same experimental conditions, was $3.80 \%$.

For interday and between analysts precision (Table 2), the absorbances obtained in three different days and by a second analyst were compared by ANOVA and F-test and $t$-test, respectively. Regarding the interday precision, the $F_{\text {calculated }}$ was lower than $F_{\text {critical }}$, and the precision between analysts $t_{\text {calculated }}$ was lower than $t_{\text {critical }}$. These data show the appropriate precision (interday and between analysists) of the method, since the absorbances obtained were statistically equivalent.

Accuracy of the method was also proved, since the recovery assay provided an average value of $100.42 \%$ (Table 3 ). This value is in accordance with the range recommended by the Horwitz Trumpet [37].

Robustness was evaluated by small variations in the following analytical conditions: temperature of the workroom, pellet compression time, and $\mathrm{KBr}$ brand. The obtained experimental values were evaluated by the $F$-test and $t$-test, by comparing the absorbance values from the normal and 
varied conditions (Table 4). For the temperature of workroom, $t_{\text {calculated }}$ was higher than $t_{\text {critical. }}$. It means the method is not robust for this aspect. In this way, the temperature of workroom must be strictly controlled for performing the method. When the air conditioning is turned off, the humidity of the environment increases, and this can be the main cause of the lack of robustness for this parameter. Regarding the other varied parameters (pellet compression time and $\mathrm{KBr}$ brand), all of them showed to be robust, since $t_{\text {calculated }}$ were lower than $t_{\text {critical }}$.

Selectivity of the method was carried out in order to verify whether the adjuvants present in the pharmaceutical dosage form were able to interfere with the analysis of amoxicillin. Figures 1 and 2 show that there are no interferences in the spectral range selected for quantitative analysis, so the selectivity was evidenced. It is important to highlight that, in Figure 2, each adjuvant was prepared in a concentration higher than that they are in the capsules, in order to better analyze the absorption bands. For this reason, the absorption bands presented in the excipient spectra are more prominent than that in Figure 1.

Three quantifications of amoxicillin in the capsules were performed, and the results are presented in Table 5. The average amoxicillin content was $100.34 \%$, which is in accordance with the range recommended by the Brazilian Pharmacopoeia (90 to 120\%) [20].

In this way, the method met all the requirements of the ICH guidelines [13] and Brazilian legislation [12] for validation of the analytical methods and can be used in quality control laboratories for the analysis of this drug. In addition, it presents low cost maintenance and is an environmentally friendly method.

\section{Conclusion}

The method has fulfilled all the requirements of the ICH guidelines and Brazilian legislation for the validation of the analytical methods. Spectrophotometry in the mid-infrared region for the quantification of amoxicillin in capsules showed adequate linearity, accuracy, precision, selectivity, and robustness for the pellet's compression time and $\mathrm{KBr}$ brand. On the other hand, the temperature of the workroom must be strictly controlled for performing the method. Therefore, this method can be used as an innovative alternative in quality control laboratories, since it is fast, clean (for the analysts and environment), presents low cost maintenance, and does not generate toxic chemical waste.

\section{Data Availability}

The data used to support the findings of this study are included within the article.

\section{Conflicts of Interest}

The authors declare that they have no conflicts of interest.

\section{Acknowledgments}

This work was supported by PACD-FCFAr-UNESP (Araraquara, Brazil), FAPESP (São Paulo, Brazil), and CNPq
(Brasília, Brazil). EG Tótoli was funded by FAPESP (São Paulo, Brazil), and HRN Salgado was funded by $\mathrm{CNPq}$ (Brasília, Brazil).

\section{References}

[1] Brazilian National Health Surveillance Agency (ANVISA), "Online directions for the use of amoxicillin," 2016, http:// www.anvisa.gov.br.

[2] H. P. Rang, M. M. Dale, J. M. Ritter, and R. J. Flower, Rang \& Dale: Farmacologia, Elsevier, Rio de Janeiro, Brazil, 6th edition, 2007.

[3] B. G. Katzung, Basic and Clinical Pharmacology, AMGH, Porto Alegre, Brazil, 10th edition, 2010.

[4] M. C. Verdier, O. Tribut, P. Tattevin, Y. Le Tulzo, C. Michelet, and D. Bentué-Ferrer, "Simultaneous determination of 12 beta-lactam antibiotics in human plasma by highperformance liquid chromatography with UV detection: application to therapeutic drug monitoring," Antimicrobial Agents and Chemotherapy, vol. 55, no. 10, pp. 4873-4879, 2011.

[5] K. Xie, M. Zhao, H. Guo et al., "Determination and depletion of amoxicillin residues in eggs," Food Additives \& Contaminants: Part A, vol. 30, no. 4, pp. 670-677, 2013.

[6] M. Szultka, R. Krzeminski, J. Szeliga, M. Jackowski, and B. Buszewski, "A new approach for antibiotic drugs determination in human plasma by liquid chromatographymass spectrometry," Journal of Chromatography A, vol. 1272, pp. 41-49, 2013.

[7] M. Szultka, R. Krzeminski, J. Szeliga, M. Jackowski, and B. Buszewski, "Simultaneous determination of selected chemotherapeutics in human whole blood by molecularly imprinted polymers coated solid phase microextraction fibers and liquid chromatography-tandem mass spectrometry," Journal of Chromatography B, vol. 940, pp. 66-76, 2013.

[8] R. R. Carzola, G. R. Romero, A. G. Frenich, M. A. Rodrigues, and J. L. Martínez, "A análise simultânea de antibióticos em amostras biológicas por espectrometria líquida de alta eficiência de ultra-massa em tandem cromatografia," Journal of Pharmaceutical and Biomedical Analysis, vol. 89, pp. 203-212, 2014.

[9] M. Carlier, V. Fogão, J. A. Roberts, E. Van De Velde, J. J. De Waele, and A. G. Verstraete, "Quantificação de sete antibióticos $\beta$-lactâmicos e dois inibidores $\beta$-lactamase em plasma humano, utilizando um método de UPLC-MS/MS validado," International Journal of Antimicrobial Agents, vol. 40, no. 5, pp. 416-422, 2012.

[10] N. E. Blazhevskiı̌, S. P. Karpova, and V. I. Kabachy̌̆, "Quantitative determination of penicillins by iodometry using potassium hydrogen peroxymonosulfate," Antibiot Khimioter, vol. 58, no. 11-12, pp. 3-7, 2013.

[11] World Health Organization, Quality Assurance of Pharmaceuticals: A Compendium of Guidelines and Related materials, Good Manufacturing Practices and Inspection, WHO, Geneva, Switzerland, 2nd edition, 2007.

[12] Brazilian Ministry of Health. Resolution of the Executive Board No. 899, Approves the Guide for Validation of Analytical and Bioanalytical Methods, Diário Oficial da União, Brasília, Brazil, 2003.

[13] ICH Q2 (R1), "Validation of analytical procedures Text and Methodology," in Proceedings of International Conference on Harmonization of Technical Requirements for the Registration of Pharmaceutical for Human Use, Geneva, Switzerland, 2005. 
[14] The United States Pharmacopeia, The National Formulary (NF 32), United States Pharmacopeia Convention, Rockville, MD, USA, 37th edition, 2014.

[15] A. M. Sanseverino, "Síntese orgânica limpa," Química Nova, vol. 23, no. 1, pp. 102-107, 2000.

[16] Association of Official Analytical Chemists, Official Methods of Analysis, AOAC, Gaithesburg, MD, USA, 17th edition, 2002.

[17] F. R. Nolasco, G. A. Tavares, and J. A. Bendassolli, "Implantação de programas de gerenciamento de resíduos químicos laboratoriais em universidades: análise crítica e recomendações," Engenharia Sanitaria e Ambiental, vol. 11, no. 2, pp. 118-124, 2006.

[18] A. H. Moreno and H. R. N. Salgado, "Development and validation of the quantitative analysis of ceftazidime in powder for injection by infrared spectroscopy," Physical Chemistry, vol. 2, no. 1, pp. 6-11, 2012.

[19] D. L. Pavia, G. M. Lampman, G. S. Kriz, and J. R. Vyvyan, Introdução à Espectroscopia, Cengage Learning, São Paulo, Brazil, 4th edition, 2010.

[20] ANVISA, Brazilian Pharmacopeia, Vol. 2, ANVISA, Brasília, Brazil, 5th edition, 2010.

[21] E. G. Tótoli and H. R. N. Salgado, "Development and validation of the quantitative analysis of ampicillin sodium in powder for injection by Fourier-transform infrared spectroscopy (FT-IR)," Physical Chemistry, vol. 2, no. 6, pp. 103-108, 2012.

[22] D. C. M. Vieira and H. R. N. Salgado, "Development and validation of the quantitative analysis of cefuroxime sodium in powder for injection by infrared spectroscopy," Advances in Analytical Chemistry, vol. 2, pp. 80-87, 2012.

[23] A. C. Kogawa and H. R. N. Salgado, "Development and validation of infrared spectroscopy method for the determination of darunavir in tablets," Physical Chemistry, vol. 33, pp. 1-6, 2013.

[24] M. S. Piantavini, R. Pontarolo, F. L. D. Pontes, C. P. Uber, D. P. Stremel, and M. M. Sena, "Chemometric quality inspection control of pyrantel pamoate, febantel and praziquantel in veterinary tablets by mid infrared spectroscopy," Spectrochimica Acta Part A: Molecular and Biomolecular Spectroscopy, vol. 125, pp. 393-403, 2014.

[25] M. A. Mallah, S. T. H. Sherazi, M. I. Bhanger, S. A. Mahesar, and M. A. Bajeer, "A rapid Fourier-transform infrared (FTIR) spectroscopic method for direct quantification of paracetamol content in solid pharmaceutical formulations," Spectrochimica Acta Part A: Molecular and Biomolecular Spectroscopy, vol. 141, pp. 64-70, 2015.

[26] L. P. Consortti and H. R. N. Salgado, "Green method for quantification of sodium cefotaxime in lyophilized powder by infrared spectroscopy," Journal of Pharmaceutical Sciences \& Emerging Drugs, vol. 5, no. 1, pp. 1-6, 2017.

[27] J. C. R. Corrêa and H. R. N. Salgado, "A platform for designing quantitative infrared spectrophotometric method for drugs and pharmaceuticals analysis: a rediscover for an ecological and safer technique in the routine quality control laboratories," World Journal of Pharmaceutical Sciences, vol. 3, no. 6, pp. 2056-2059, 2014.

[28] A. C. Kogawa, F. A. Aguiar, C. M. Gaitani, and H. R. N. Salgado, "Validation of a stability indicating capillary electrophoresis method for the determination of darunavir in tablets and comparison with the of infrared absorption spectroscopic method," World Journal of Pharmaceutical Sciences, vol. 3, pp. 283-297, 2014.
[29] A. C. Kogawa, N. P. Mello, and H. R. N. Salgado, "Quantification of doxycycline in raw material by an eco-friendly method of infrared spectroscopy," Pharmaceutica Analytica Acta, vol. 7, no. 2, pp. 463-466, 2016.

[30] A. C. Kogawa and H. R. N. Salgado, "Spectrophotometry in infrared region: a new, low cost and green way to analyze tablets of rifaximin," Current Pharmaceutical Analysis, vol. 14, no. 2, pp. 108-111, 2018.

[31] A. C. Kogawa and H. R. N. Salgado, "Impact of rifaximin potency after treatment with different solvents," Journal of Pharmaceutical and Biological Sciences, vol. 5, pp. 77-80, 2017.

[32] A. C. Kogawa and H. R. N. Salgado, "Rifaximin stability: a look by UV, IR, HPLC and turbidimetry method," Journal of AOAC International, vol. 101, no. 2, pp. 410-413, 2018.

[33] B. A. Marco and H. R. N. Salgado, "Development and validation of an innovative method for the determination of cefadroxil monohydrate in capsules," Physical Chemistry, vol. 6, pp. 67-74, 2016.

[34] J. S. H. Natori, E. G. Tótoli, and H. R. N. Salgado, "Development and validation of a green analytical method for determination of norfloxacin in raw material by FourierTransform Infrared Spectrophotometry (FT-IR)," Journal of AOAC International, vol. 99, no. 6, pp. 1533-1536, 2016.

[35] E. G. Tótoli and H. R. N. Salgado, "Fourier-Transform Infrared (FT-IR) spectrophotometry: an eco-friendly method for the analysis of daptomycin injectable," Journal of AOAC International, vol. 100, no. 5, pp. 1569-1576, 2017.

[36] M. T. Trindade and H. R. N. Salgado, "Development and validation of a modern and stability-indicating method for the quantification of ceftriaxona sodium in powder for injection by infrared spectroscopy," Physical Chemistry, vol. 7, pp. 55-62, 2017.

[37] M. Valcárcel, Principles of Analytical Chemistry: A Textbook, Springer, Berlin Heidelberg, Germany, 6th edition, 2000. 

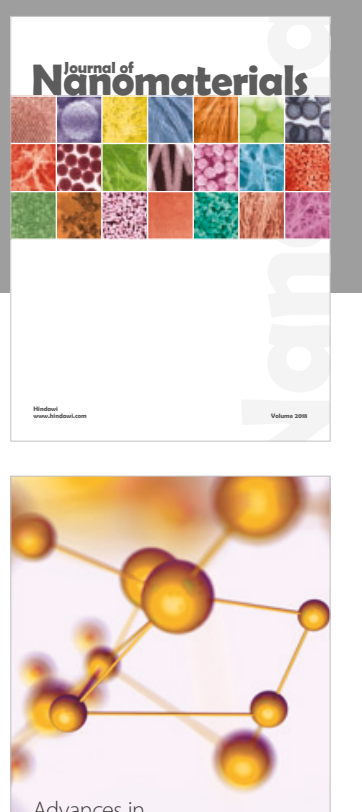

Physical Chemistry
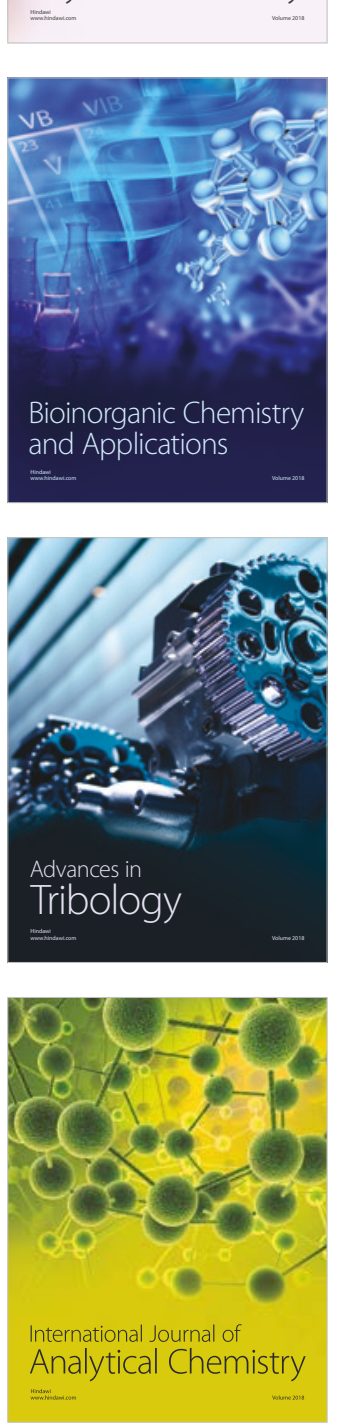

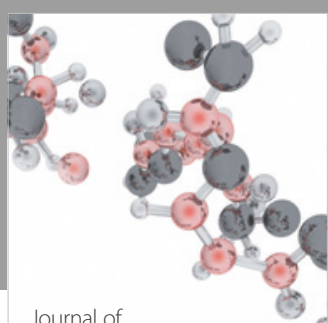

Analytical Methods

in Chemistry

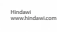

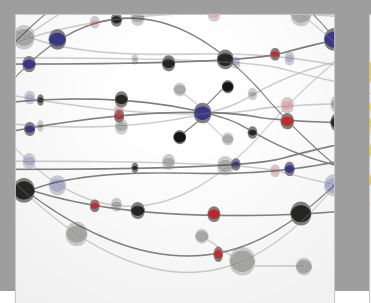

The Scientific World Journal

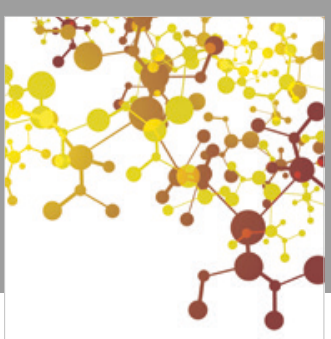

Journal of

Applied Chemistry
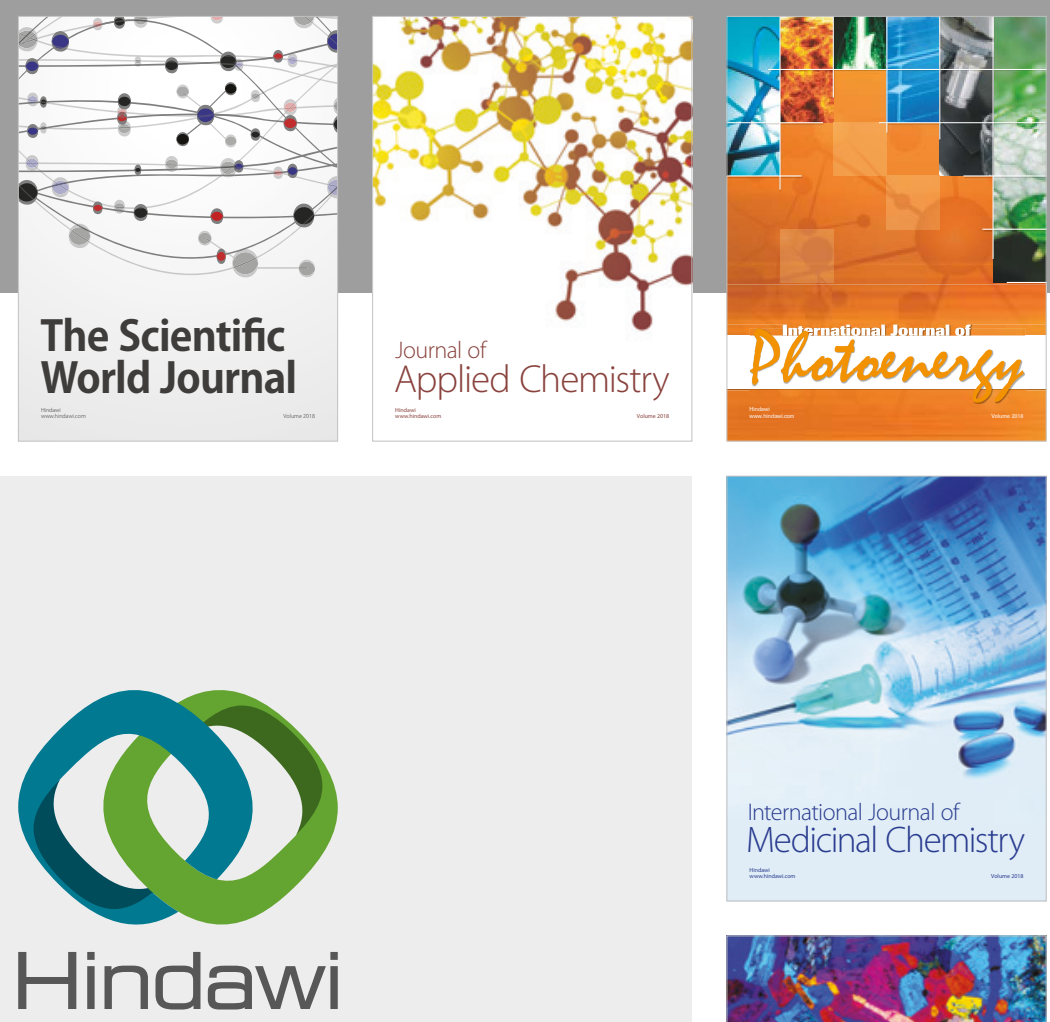

Submit your manuscripts at

www.hindawi.com
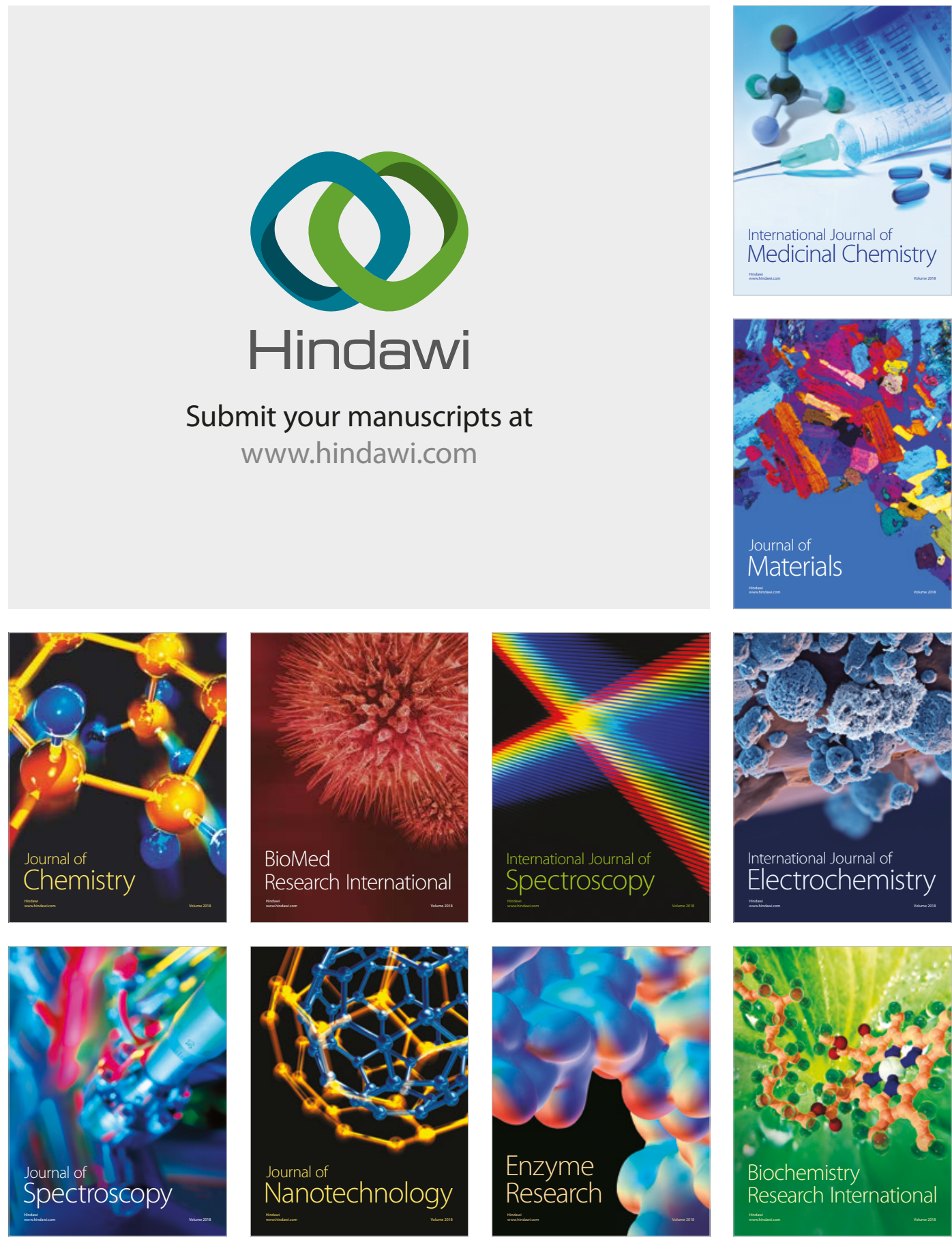
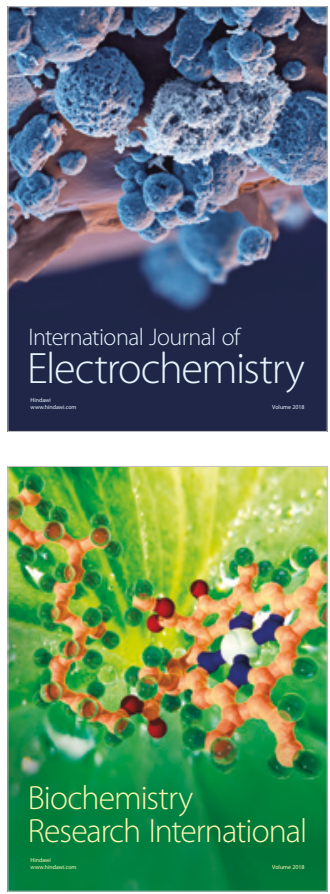